\title{
A fast algorithm for the simulation of arterial pulse waves
}

\author{
Tao $\mathrm{Du}^{\mathrm{a}}$, Dan $\mathrm{Hu}^{\mathrm{a}, *}$, David Cai ${ }^{\mathrm{a}, \mathrm{b}, \mathrm{c}}$ \\ ${ }^{a}$ Department of Mathematics, Institute of Natural Sciences, and MOE-LSC, \\ Shanghai Jiao Tong University, Shanghai, China \\ ${ }^{b}$ Courant Institute of Mathematical Sciences and Center for Neural Science, \\ New York University, New York, U.S.A. \\ ${ }^{c}$ NYUAD Institute, New York University Abu Dhabi, PO Box 129188, Abu Dhabi, UAE
}

\begin{abstract}
One-dimensional models have been widely used in studies of the propagation of blood pulse waves in large arterial trees. Under a periodic driving of the heartbeat, traditional numerical methods, such as the Lax-Wendroff method, are employed to obtain asymptotic periodic solutions at large times. However, these methods are severely constrained by the CFL condition due to large pulse wave speed. In this work, we develop a new numerical algorithm to overcome this constraint. First, we reformulate the model system of pulse wave propagation using a set of Riemann variables and derive a new form of boundary conditions at the inlet, the outlets, and the bifurcation points of the arterial tree. The new form of the boundary conditions enables us to design a convergent iterative method to enforce the boundary conditions. Then, after exchanging the spatial and temporal coordinates of the model system, we apply the Lax-Wendroff method in the exchanged coordinate system, which turns the large pulse wave speed from a liability to a benefit, to solve the wave equation in each artery of the model arterial system. Our numerical studies show that our new algorithm is stable and can perform $\sim 15$ times faster than the traditional implementation of the Lax-Wendroff method under the requirement that the relative numerical
\end{abstract}

\footnotetext{
*I am corresponding author.

Email addresses: duty1998@sjtu.edu.cn (Tao Du), hudan80@sjtu.edu.cn (Dan Hu ), cai@cims.nyu.edu (David Cai)
}

Preprint submitted to Journal of Computational Physics

January 25, 2016

(C) 2016. This manuscript version is made available under the Elsevier user license http://www.elsevier.com/open-access/userlicense/1.0/ 
error of blood pressure be smaller than one percent, which is much smaller than the modeling error.

Keywords: Blood pulse wave, Riemann variables, Lax-Wendroff method, large wave speed, fast algorithm

\section{Introduction}

In Traditional Chinese medicine, the temporal profile of blood pressure is believed to be a useful indicator of an individual's physiological condition [1, 2] . Strong correlations have been observed between the blood pulse profiles and diseases, such as hypertension [3 5], arteriosclerosis [6, 7], and diabetes mellitus 8]. Certain blood pulse profiles in these diseases are believed to be resulted from abnormality in geometric structures and elastic properties of the arterial system 9 13]. In computational modeling, one-dimensional models have been shown to be able to capture the propagation of blood pulse waves and the profile of the blood pressure in large arterial trees [14 28]. The computational modeling has provided an important approach to the study of relations between abnormal blood pressure profiles and diseases [22, 23, 26, 29].

In many applications, a large amount of simulations of the one-dimensional model may be necessary. For example, different diseases can lead to different types and varying degrees of alterations in the geometric structures and elastic properties of the arterial tree. To study the relation between these changes and the resulting blood pulse profiles [22, 23, 26, 29], there is a need to use a large number of numerical simulations to explore all possible dynamics of the arterial tree brought about by those changes; In general, it is difficult to obtain all detailed information of the physical properties of the arterial tree as well as the inflow information at the heart. To quantify this type of uncertainty of dynamical models and the accuracy of computed physiological properties in virtual populations, it is also necessary to perform a great number of simulations with a broad range of parameters [21, 30, 31]; Furthermore, following the idea of the Traditional Chinese medicine, the inverse problem of gauging the blood 
pulse profiles to apprehend clinical changes associated with properties of the arterial system is of particular interest. To tackle this problem successfully, it would also entail a large number of simulations of the forward problem. Clearly, a fast algorithm for solving the one-dimensional model would greatly facilitate these studies.

Traditional numerical methods, such as the Lax-Wendroff method [32], are widely used in solving the one-dimensional model 15, 29, 33 37]. However, due to the large speed of blood pulse waves $\left(e . g ., \sim 1 \times 10^{3} \mathrm{~cm} / \mathrm{s}\right)$ and short lengths of vessels (e.g., the length of hepatic artery is $\sim 1 \mathrm{~cm}$, see Table 4) [5, 38], the time step required by the CFL condition [39] for the Lax-Wendroff method is usually very small $\left(e . g ., \sim 10^{-4} s\right)$. The requirement of small time step by the CFL condition also presents a strong constraint in the implementation of other methods for solving the one-dimensional model, including the MacCormack scheme 28, 40], the upwind scheme [41], the finite element method [24, 25], the Galerkin method 20, 20 22, 26], and the domain-decomposition method 27]. Furthermore, because the heartbeat is approximately periodic, one usually seeks periodic solutions of the one-dimensional model for blood pulse waves. This often requires one to evolve the model for sufficiently long time ( $\sim 10$ periods) to obtain an asymptotic periodic solution. As demanded by the small time step and long simulation time, the simulation can become rather expensive.

In this work, we develop a fast algorithm for the simulation of the onedimensional model of blood pulse waves. First, we reformulate the model in terms of a set of Riemann variables. Then we exchange the temporal and spatial coordinates and apply the Lax-Wendroff method in the exchanged coordinate system to solve the wave equations in each vessel. Finally, in order to enforce the boundary conditions at the inlet, the outlets, and the bifurcation points of the arterial tree, we derive a new form of the boundary conditions, which allows us to design a stable iterative method. The new algorithm can overcome the constraint of the CFL condition in the case of large wave speed. In fact, in our algorithm, the large wave speed is no longer a liability — instead, it has become an advantage. As a result, the computational cost of our algorithm reduces 
significantly. Under the requirement that the numerical error of blood pressure be smaller than one percent, which is already much smaller than the error of the model, our algorithm yields $\sim 15$ times of speedup compared to the traditional scheme [15, 33].

\section{One-dimensional model for arterial pulse waves}

The propagation of blood pulse waves in an arterial tree includes two processes - the interaction between the blood flow and the elastic vessel walls in single vessels and the wave transmission and reflection at the vessel bifurcation points (or junctions). The first one is described by a system of partial differential equations and the second one is described by the boundary conditions at the bifurcations of the arterial tree. The one-dimensional model for blood pulse waves has been studied in the previous works [4, 5, 15, 16, 19, 42]. In this section, we briefly review the model and reformulate the system using two sets of Riemann variables, which are used to facilitate the design of our algorithm.

2.1. Blood pulse wave in a single vessel

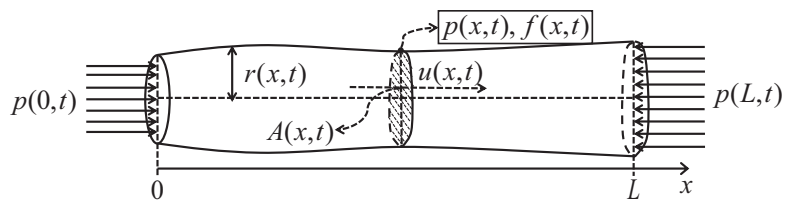

Figure 1: An axisymmetric vessel with radius $r(x, t)$ and fixed length $L$. The direction of the average blood velocity $u(x, t)$ is along the axis of the vessel. The viscous effect $f(x, t)$ arises from the shear stress on the vessel wall.

As is in the previous works [4, 5, 15, 16, 19, 42], a blood vessel is regarded as an axisymmetric compliant tube with fixed length $L$ and variable radius $r(x, t)$, where $t$ is the temporal coordinate, $x$ is the spatial coordinate along the central axis of the vessel, as shown in Fig. 1. The blood is assumed to be 
an incompressible Newtonian fluid. Under long wavelength approximation, the conservation of momentum and mass of the blood flow in the tube yields

$$
\begin{gathered}
u_{t}+u u_{x}+\frac{p_{x}}{\rho}=\frac{f}{\rho}, \\
A_{t}+A u_{x}+u A_{x}=0,
\end{gathered}
$$

where $u(x, t)$ is the average blood velocity over the cross section at $x, p(x, t)$ is the average pressure on the cross section, $\rho$ is the constant density of the blood, $A(x, t)=\pi r(x, t)^{2}$ is the cross-sectional area, and $f(x, t)$ represents the viscous effect on the vessel wall

$$
f(x, t)=\frac{2 \pi r(x, t)}{A(x, t)} \tau(x, t),
$$

where $\tau(x, t)$ is the axisymmetric wall shear stress.

Since there are four unknowns $(u, p, A$, and $\tau)$ but only two equations in the model (1), two additional constitutive equations are needed to close the system. We discuss this closure in the following.

First, in one-dimensional modeling, the arterial wall of a vessel is usually assumed to be thin, isotropic, homogeneous, incompressible, and purely elastic. It is further assumed that the deformation is axisymmetric within each circular cross section. This leads to a relation between the blood pressure and the crosssectional area [16, 17, 33, 43, 44]. In general, the deformation is small and linear elasticity is applicable. Under this approximation, we have

$$
p(A)=\frac{1}{2} \rho c_{0}^{2}\left(\left(\frac{A}{A_{0}}\right)^{2}-1\right)+p_{0},
$$

where $A_{0}(x)$ and $r_{0}(x)$ are the reference cross-sectional area and radius, respectively, when the blood pressure $p$ is equal to the tissue pressure $p_{0}$. The reference pulse wave speed, $c_{0}=\sqrt{\frac{2 E h}{3 \rho r_{0}(x)}}$, is the limiting speed of the pulse wave in the vessel as $A \rightarrow A_{0}$, where $E$ is the Young's modulus and $h$ is the thickness of the arterial wall [15].

Second, since we are studying flows under a pulsatile drive, the Womersley model [45] is used to obtain the profile of the blood velocity in the cross section and to calculate the shear stress on the vessel wall [15]. The velocity profile 
of the blood velocity in a cross section is mainly determined by the Womersley number, $W=r_{0} \sqrt{\omega \rho / \mu}$, where $\omega$ is the characteristic frequency and $\mu$ is the blood viscosity. When the Womersley number is small (e.g., $W<3$ for low frequency and small vessels), the Womersley velocity profile is nearly parabolic, similar to the Poiseuille flow; When the Womersley number is large (e.g., $W>10$ for high frequency or large vessels. The Womersley number in the aorta for the fundamental frequency corresponding to a heartbeat period is 13), the Womersley model can capture the effect of the boundary layer arising from the pulsatile blood flow in large vessels [45, 46]. Under the Womersley approximation, the Fourier modes of the wall shear stress, $\tau(x, t)$, is given by

$$
\widehat{\tau}(x, \omega)= \begin{cases}-\frac{i \rho \omega \widehat{Q}(x, \omega) r_{0} F_{J}}{2 A_{0}\left(1-F_{J}\right)} & \text { for } \omega \neq 0, \\ -\frac{4 \pi \mu r_{0}}{A_{0}^{2}} \widehat{Q}(x, 0) & \text { for } \omega=0,\end{cases}
$$

where $\widehat{Q}(x, \omega)$ is the Fourier mode of the blood flow rate $Q(x, t)=u A$ (Throughout the text, we use the notation $\widehat{X}(\omega)$ to stand for the Fourier transform of the variable $X(t))$. Here $F_{J}=\frac{2 J_{1}\left(h_{0}\right)}{h_{0} J_{0}\left(h_{0}\right)}, h_{0}=\sqrt{-i} W$, and $J_{0}(\cdot)$ and $J_{1}(\cdot)$ are the zeroth and first order Bessel functions of the first kind, respectively. Under the assumptions of linear elasticity and the Womersley velocity profile, the one-dimensional model becomes

$$
\begin{aligned}
u_{t}+u u_{x}+c^{2} \frac{A_{x}}{A} & =\frac{f}{\rho}, \\
A_{t}+A u_{x}+u A_{x} & =0,
\end{aligned}
$$

where $c=\frac{A}{A_{0}} c_{0}$ is the pulse wave speed.

In our work, the thickness of the vessel wall is assumed to satisfy $h=$ $c_{1}\left(r_{0} / r_{\mathrm{f}}\right)^{c_{2}}$ [47, 48], where $r_{\mathrm{f}}=1 \mathrm{~cm}$ and the parameters $c_{1}=0.1204 \mathrm{~cm}$ and $c_{2}=0.6244$ were fitted with the experimental data in Ref. [49, 50]. As a result of the difference in the relative amount of collagen and elastin in arterial walls, the Young's modulus of arterial walls increases as the arterial radius decreases [9, 51, 52]. As in the previous work [38], the Young's modulus is assumed to satisfy the relation $E=k_{1} e^{k_{2} r_{0}}+k_{3}$, where $k_{1}=2.055 \times 10^{7} \mathrm{~g} /\left(\mathrm{s}^{2} \mathrm{~cm}\right)$, 
$k_{2}=-5.634 \mathrm{~cm}^{-1}$, and $k_{3}=4.182 \times 10^{6} \mathrm{~g} /\left(\mathrm{s}^{2} \mathrm{~cm}\right)$ were fitted with data from the work of Ref [5]. The dynamical blood viscosity is $\mu / \rho=0.046 \mathrm{~cm}^{2} / \mathrm{s}$ [33, 44].

Based on these data, the reference pulse wave speed, $c_{0}=\sqrt{\frac{2 E h}{3 \rho r_{0}(x)}}$, is approximately $5.4 \times 10^{2} \mathrm{~cm} / \mathrm{s}$ in the ascending aorta with a radius of $1.44 \mathrm{~cm}$. As the vessel radius decreases, the pulse wave speed increases since both the Young's modulus and the wall-to-lumen ratio, $\frac{h}{r_{0}}$, increases. For example, the pulse wave speed is approximately $1.8 \times 10^{3} \mathrm{~cm} / \mathrm{s}$ in a vessel with a radius of $0.091 \mathrm{~cm}$. We comment that arterial thickening and stiffening can lead to a significant increase of the pulse wave speed. For example, the typical aortic pulse wave speed can be as large as $1.2 \times 10^{3} \mathrm{~cm} / \mathrm{s}$ in the old people [9].

\subsection{Riemann variables}

The method of Riemann variables is a useful tool for studying wave equations. For the system (5), the characteristic lines are $\frac{d x}{d t}=u \pm c$ and the corresponding Riemann variables $\Re^{ \pm}(x(t), t)=u \pm c$ satisfy

$$
\frac{d \Re^{ \pm}}{d t}=\frac{\partial \Re^{ \pm}}{\partial t}+(u \pm c) \frac{\partial \Re^{ \pm}}{\partial x}=\frac{f}{\rho}
$$

on the characteristic lines. In the aorta, the typical flow velocity is $\sim 20 \mathrm{~cm} / \mathrm{s}$ and the vibrational amplitude of the blood pressure is $\sim 20 \mathrm{mmHg}[15$, , 38]. Thus we have approximately $\left|\frac{u}{\bar{c}}\right|<5 \%$ and $\left|\frac{c-\bar{c}}{\bar{c}}\right|<8 \%$ in the ascending aorta, where $\bar{c}$ is the average pulse wave speed in the vessel. These small ratios enable us to approximate the system (6) by the linear system

$$
\frac{\partial \Re^{ \pm}}{\partial t} \pm \bar{c} \frac{\partial \Re^{ \pm}}{\partial x}=\frac{f}{\rho},
$$

because the nonlinearity of the system (6) is weak. In downstream arteries, these ratios become even smaller because, with decreasing vessel radius, the flow velocity $u$ decreases and the pulse wave speed increases. As a result, for an arterial crown with small root-vessel radius (e.g., smaller than $1.5 \mathrm{~mm}[53]$ ), the nonlinearity is negligible. As a consequence of this weak nonlinearity and the short duration of time for the pulse wave to propagate through the arterial system, there would be no formation of shock waves in the arterial system. 
In the previous works [15, 33], another set of Riemann variables, $£^{ \pm}$, is defined as

$$
£^{ \pm}(x, t)=p(x, t) \pm R_{1} Q(x, t),
$$

where $R_{1}=\frac{\rho c_{0}}{A_{0}}$ is the characteristic resistance of the vessel [4, 53]. Using $£^{ \pm}$, we can also rewrite Eq. (5D) as

$$
\frac{\partial £^{ \pm}}{\partial t}+(u \pm c) \frac{\partial £^{ \pm}}{\partial x}= \pm c f+u\left(p_{x} \mp \rho c u_{x}\right) .
$$

Note that the flow velocity $u$ is much smaller than the pulse wave speed $c$. The second term on the right hand side of Eq. (9) is small compared to the second term on the left hand side.

In our new numerical algorithm, $\Re^{ \pm}$is used in solving Eq. (5) in the vessels and $£^{ \pm}$is used in dealing with the boundary conditions at the outlets and the bifurcation points. The two sets of Riemann variables are related to each other through the following relations

$$
\begin{aligned}
& A=\frac{A_{0}\left(\Re^{+}-\Re^{-}\right)}{2 c_{0}}, \\
& u=\frac{\Re^{+}+\Re^{-}}{2}, \\
& p=\frac{1}{2} \rho c_{0}^{2}\left(\left(\frac{A}{A_{0}}\right)^{2}-1\right)+p_{0}, \\
& Q=u A \text {, } \\
& £^{ \pm}=p \pm R_{1} Q, \\
& \begin{aligned}
p & =\frac{£^{+}+£^{-}}{2}, \\
Q & =\frac{£^{+}-£^{-}}{2 R_{1}}, \\
A & =A_{0} \sqrt{\frac{2\left(p-p_{0}\right)}{\rho c_{0}^{2}}+1},
\end{aligned} \\
& u=\frac{Q}{A}, \\
& \Re^{ \pm}=u \pm \frac{A c_{0}}{A_{0}} .
\end{aligned}
$$

\subsection{Boundary conditions}

Boundary conditions of the one-dimensional model have been discussed in the previous works 1428,33 , 53]. In the following, we review the boundary conditions and formulate them with the Riemann variables, $\Re^{ \pm}$(at the inlet) or $£^{ \pm}$(at the bifurcations and outlets). We will take advantage of the new formulation involving the Riemann variables in our numerical method.

Inlet boundary condition

The cardiac output $q_{c}(t)$, which is the blood flow rate pumped into the aorta by the heart and can usually be measured experimentally, is used to determine 
the boundary condition at the inlet of an arterial tree [15, 33, 53],

$$
Q_{\text {root }}(0, t)=q_{c}(t)
$$

where the subscript "root" indicates the root vessel. We use $x=0$ and $x=L$ to represent the inlet and outlet of a vessel, respectively. From the expressions in Eq. (10), the Riemann variables $\Re_{\text {root }}^{ \pm}(0, t)$ at the inlet of the root vessel satisfy $\left[\Re_{\text {root }}^{+}(0, t)\right]^{2}-\left[\Re_{\text {root }}^{-}(0, t)\right]^{2}=\frac{4 c_{0, \text { root }} q_{c}(t)}{A_{0, \text { root }}}$. Thus we have

$$
\Re_{\text {root }}^{+}(0, t)=\sqrt{\left[\Re_{\text {root }}^{-}(0, t)\right]^{2}+\frac{4 c_{0, \text { root }} q_{c}(t)}{A_{0, \text { root }}}},
$$

which can be used to update $\Re_{\text {root }}^{+}(0, t)$ with given $\Re_{\text {root }}^{-}(0, t)$ at the inlet in our numerical algorithm.

\section{Outlet boundary conditions}

In general, there are too many small peripheral vessels in the arterial tree to be incorporated in the model. It is necessary to truncate small arterial crowns (the sub-tree of the arterial tree) in the simulation. An arterial tree and the truncated vessel crowns are illustrated in Fig. 2. At the truncation points, boundary conditions are deployed to represent the effects of the truncated vessel crowns.

As discussed above, the nonlinearity of the model (5) is negligible for small vessel crowns. The boundary condition at an outlet can therefore be described by a linear relation between the blood pressure and the blood flow rate, with the following form in the Fourier space [15, 44, 53.

$$
\widehat{p}(L, \omega)=\widehat{Q}(L, \omega) Z(\omega),
$$

where $Z(\omega)$ is the impedance of the truncated vessel crown. For the blood pulse wave in a large arterial tree, the net effect of a small vessel crown is to generate a reflection wave for an incident wave. Using Eq. (8), we can reformulate the boundary condition (13) as

$$
\widehat{£^{-}}(L, \omega)=\widehat{£^{+}}(L, \omega) \frac{Z(\omega)-R_{1}}{Z(\omega)+R_{1}},
$$




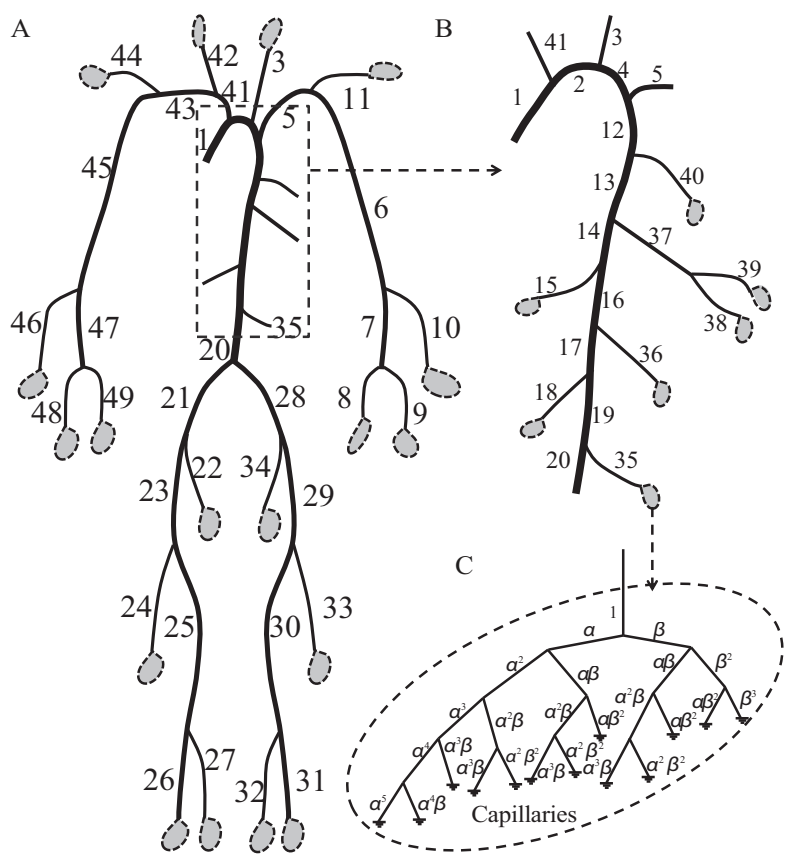

Figure 2: The systemic arterial tree and the truncated vessel crowns. A: the main human systemic arterial tree; B: details of the principal aortic branches. The indices in A and B, as tabulated in Table 1, indicate specific arteries. Gray ellipsoids attached to the arterial tree in A and B represent truncated arterial crowns; C: a representative truncated arterial crown modeled by a structured tree. The parameters $\alpha$ and $\beta$ are the bifurcation ratios in a structured tree model (see text).

which can be viewed as the linear reflection rule for each component of the wave reflected by the truncated vessel crown with the factor,

$$
R_{\mathrm{f}}=\frac{Z(\omega)-R_{1}}{Z(\omega)+R_{1}},
$$

as the reflection coefficient.

We now turn to the discussion of how the reflection coefficient is computed in our work. We also describe the properties of the reflection coefficient and the role it plays in our numerical algorithm.

We use structured trees, introduced in Ref. [15, 33, 38, 44], to model the truncated arterial crowns. In a structured tree, as shown in Fig. $2 \mathrm{C}$, the radii of the left- and the right-daughter vessels are given by $R_{l}=\alpha R_{p}$ and $R_{r}=\beta R_{p}$, 
respectively, where $R_{p}$ is the radius of the parent vessel. As in the previous works [15, 33, 38, 44], we assume that the bifurcation ratios $\alpha=0.9$ and $\beta=0.6$ and the length of a vessel is proportional to its radius, $L=50 R$.

When the radii of all the vessels in an arterial crown are small, the linear system (7) can be used to describe the wave propagation. The impedance of an arterial crown modeled by a structured tree can be calculated by the following recursive steps [15, 33, 38, 44]. Step one, using the Fourier transform of Eq. (77), the impedance at the inlet $(x=0)$ of a vessel can be obtained from the impedance at the outlet $(x=L)$

$$
Z(0, \omega)=\frac{\lambda}{i \omega C} \frac{i \omega C Z(L, \omega) \cosh (\lambda L)+\lambda \sinh (\lambda L)}{\lambda \cosh (\lambda L)+i \omega C \sinh (\lambda L) Z(L, \omega)},
$$

where $\lambda=\sqrt{\frac{-\rho C \omega^{2}}{A_{0}\left(1-F_{J}\right)}}$. The real and imaginary parts of $\lambda$ are the spatial decay rate and the wave number of the wave, respectively. Step two, as in an electrical circuit of two parallel resistors, the total impedance of two parallel subtrees is

$$
Z_{P}(L, \omega)=\frac{Z_{L}(0, \omega) Z_{R}(0, \omega)}{Z_{L}(0, \omega)+Z_{R}(0, \omega)}
$$

where $Z_{P}(L, \omega), Z_{L}(0, \omega)$, and $Z_{R}(0, \omega)$ are the impedances of the parent, the left-, and the right-daughter vessels at the bifurcation point, respectively. Setting the impedance at the distal ends of an arterial crown to be zero, equations (16) and (17) can be used recursively to calculate the impedance of the full arterial crown, i.e., $Z(\omega)$ in Eq. (13).

At each truncation point of the large arterial tree, we attach a structured tree as illustrated in Fig. 2, Then we use the above recursive steps to calculate the linear impedance, thus the linear reflection coefficient (Eq. (15)), at all the outlets of the large arterial tree. In the left panel of Fig. 3. we plot the linear reflection ratio, $\left|R_{\mathrm{f}}\right|$, and the phase shift, $\Phi\left(R_{\mathrm{f}}\right)$, at the outlet of a representative small vessel (vessel 48 in Fig. 2). $\left|R_{\mathrm{f}}\right|$ and $\Phi\left(R_{\mathrm{f}}\right)$ are the magnitude and the phase angle of the reflection coefficient, respectively. As is expected, from Fig. 3 , it can be seen that the linear reflection ratio is always smaller than 1 , indicating a reduction in the amplitude of the reflected wave relative to the incident wave. We note that there is in general a strong reduction at high frequencies. This 
reduction provides us with a contraction property, which will be exploited below in designing the iteration method in our numerical algorithm.
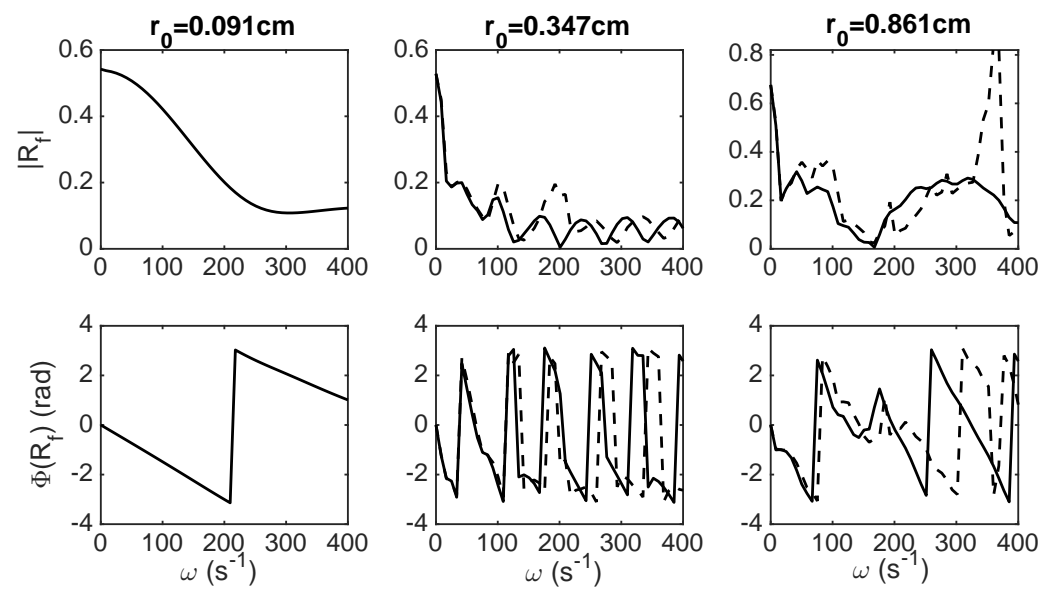

Figure 3: The amplitude, $\left|R_{\mathrm{f}}\right|$, and the phase angle, $\Phi\left(R_{\mathrm{f}}\right)$, of the linear and nonlinear reflection coefficient. Reflection coefficient is calculated by Eq. [15), in which the impedance for the linear reflection ratio (solid lines) is obtained from the recursive procedure whereas that for the nonlinear reflection coefficient (dashed lines) is calculated by Eq. 20] using data from our full simulation of one-dimensional model of the arterial tree shown in Fig. 2/A. The left, middle, and right panels correspond to the vessels with index $48\left(r_{0}=0.091 \mathrm{~cm}\right), 23$ $\left(r_{0}=0.347 \mathrm{~cm}\right)$, and $14\left(r_{0}=0.861 \mathrm{~cm}\right)$, respectively. The large jumps in the phase angle arise naturally from period reset since the phase angle is in the interval $[-\pi, \pi)$.

\section{Boundary conditions at bifurcation points}

At a bifurcation point of an arterial tree, the conservation of mass and the continuity of pressure are used to determine the boundary conditions [15, 38, 53],

$$
Q_{p}=Q_{l}+Q_{r} \text { and } p_{p}=p_{l}=p_{r}
$$

where the subscripts $p, l$, and $r$ are used throughout the text to denote the parent, the left-, and the right-daughter vessels, respectively. Using the Riemann variables, $£^{ \pm}$, we can reformulate the boundary conditions as a linear combination of the waves propagating toward and away from the bifurcation point in 
the three vessels. The new boundary conditions now read

$$
\left(\begin{array}{c}
£_{p}^{-}\left(L_{p}, t\right) \\
£_{l}^{+}(0, t) \\
£_{r}^{+}(0, t)
\end{array}\right)=D\left(\begin{array}{c}
£_{p}^{+}\left(L_{p}, t\right) \\
£_{l}^{-}(0, t) \\
£_{r}^{-}(0, t)
\end{array}\right),
$$

where $L_{p}$ is the lenghth of the parent vessel and the constant matrix $D$ has the form

$$
D=\left(\begin{array}{ccc}
2 \alpha_{p}-1 & 2 \alpha_{l} & 2 \alpha_{r} \\
2 \alpha_{p} & 2 \alpha_{l}-1 & 2 \alpha_{r} \\
2 \alpha_{p} & 2 \alpha_{l} & 2 \alpha_{r}-1
\end{array}\right),
$$

where $\alpha_{i}=\frac{C_{1 i}}{C_{1 p}+C_{1 r}+C_{1 l}},(i=p, l$, or $r)$ and $C_{1 i}=\frac{A_{0 i}}{\rho c_{0 i}}$ is the reciprocal of the characteristic resistance, $R_{1 i}$, of vessel $i$. As $D$ is a constant matrix, Eq. (18) can be represented in either the time domain or the frequency domain.

In an iteration algorithm, Eq. (18) can be used to update the three waves on the left hand side when given waves on the right hand side. The sum of all the elements in each row in $D$ is 1 . However, the sum of their absolute values is in general greater than 1. As a result, a repeated use of Eq. (18) in the iteration can cumulatively lead to an amplification of numerical error in the sense of $L^{\infty}$ norm. In order to have a desired contraction property for our algorithm, we derive a nonlinear reflection rule similar to Eq. (14).

Using the Riemann variables $£^{ \pm}(x, \omega)$, we can define the wave-dependent impedance of the vessel crown downstream with respect to a spatial point $x$

$$
Z(x, \omega)=\frac{\hat{Q}(x, \omega)}{\hat{p}(x, \omega)}=\frac{£^{+}(x, \omega)+£^{-}(x, \omega)}{£^{+}(x, \omega)-£^{-}(x, \omega)} R_{1} .
$$

Again similar to an electrical circuit of two parallel resistors, the total impedance of the parallel system of the left- and the right-daughter vessel crowns is given by

$$
\frac{1}{Z_{p}\left(L_{p}, \omega\right)}=\frac{£_{l}^{+}(0, \omega)-£_{l}^{-}(0, \omega)}{R_{1 l}\left(£_{l}^{+}(0, \omega)+£_{l}^{-}(0, \omega)\right)}+\frac{£_{r}^{+}(0, \omega)-£_{r}^{-}(0, \omega)}{R_{1 r}\left(£_{r}^{+}(0, \omega)+£_{r}^{-}(0, \omega)\right)} .
$$

Combining Eqs.(18) and (20), we arrive at the following boundary conditions 
at a bifurcation point:

$$
£_{p}^{-}\left(L_{p}, \omega\right)=£_{p}^{+}\left(L_{p}, \omega\right) \frac{Z_{p}\left(L_{p}, \omega\right)-R_{1 p}}{Z_{p}\left(L_{p}, \omega\right)+R_{1 p}},
$$

where the factor $\frac{Z_{p}\left(L_{p}, \omega\right)-R_{1 p}}{Z_{p}\left(L_{p}, \omega\right)+R_{1 p}}$ can be naturally viewed as a reflection coefficient — which is a nonlinear one since it depends on $£_{p}^{+}$. The second and third components of Eq. (18) and the nonlinear reflection rule (21) will be used in our iteration algorithm below to update the variables at bifurcation points.

We now discuss the properties of nonlinear reflection coefficient. In Fig. 3 . we display the reflection ratio and phase of the linear and nonlinear reflection coefficients. The linear reflection coefficient is obtained by the recursive procedure described above. We comment that the vessels in a large arterial tree have large radii and the linear reflection coefficient is not accurate in describing the reflection rule for large arteries. For two large vessels of radii $0.861 \mathrm{~cm}$ and $0.347 \mathrm{~cm}$, we display the linear reflection coefficients for comparison with the nonlinear ones in the left and middle panels of Fig. 3. The nonlinear reflection coefficient is obtained using Eq. (20) with data from the full simulation of our one dimensional model of the blood pulse wave in the large arterial tree as shown in Fig. 2A. As can be seen from the difference between the linear and nonlinear reflection ratios shown in Fig. 3, albeit weak, the nonlinearity cannot be neglected in large arteries. In the linear combination rule (18), the phase difference between the Riemann variables plays an important role in determining the reflected wave. Because small vessel crowns are usually short, the time lag between an incident wave into a small vessel crown and the reflection wave in the vessel crown is small. As a result, the reflection ratio shown in Fig. 3 is smoother (the left and middle panels) for the small artery than that for the large one (the right panel).

As depicted in Fig. 3, the nonlinear reflection ratio is also smaller than 1. We will make use of this reduction in amplitude, which gives rise to the aforementioned desired contraction property in our iteration method. Note that the nonlinear reflection ratio depends not only on the vessel tree structure, but also on the incident wave. For example, when the incident wave is a monochromatic 
wave, the reflection wave is not monochromatic due to nonlinearity. Therefore, the reflection coefficient obtained from Eq. (20) may be infinite at certain frequencies. However, in general, a physiological incident wave has a broad frequency band with strong low frequency components. For the physiological range of parameters, over a broad range of frequency, the additional contribution due to nonlinearity in the reflection wave is small compared to the magnitude of the incident wave for a broad range of frequency. Consequently, the contraction property is always maintained in our simulation. For extremely high frequencies, in practice, we could simply set the high frequency components of the reflection coefficient to be zero had the reflection ratio ever become greater than 1 . However, we have never encountered with this situation in our simulations.

\section{Periodic boundary conditions in time}

As mentioned above that the heartbeat is approximately periodic, the blood pulse wave therefore also behaves approximately periodically as well. The periodic boundary condition in time can be naturally imposed as

$$
\Re^{ \pm}(x, 0)=\Re^{ \pm}(x, T)
$$

to obtain periodic solutions, where $T$ is the period of heartbeat.

\section{The fast algorithm for blood waves in large arterial trees}

\subsection{Bottleneck of the traditional methods}

As discussed previously, although the model for arterial pulse waves is onedimensional, there are a few factors that can render the simulation expensive: First, there are a large number of vessels in the arterial tree even after the truncation of vessel crowns; Second, due to the small length $(e . g ., 1 \mathrm{~cm})$ of some vessels and the large pulse wave speed, the time step in the simulation must be set to be sufficiently small to satisfy the CFL condition. For example, oftentimes a time step of $\sim 10^{-4} s$ is used, which is very small comparing to the period of a heartbeat. For simulating one-dimensional arterial pulse waves, the small 
time step is a common bottleneck in the traditional methods, ranging from finite difference methods to finite element methods [5, 15, 20 22, 24 29, $33-$ 38, 40, 41]. Finally, in order to obtain an approximate periodic solution, one needs to evolve the system for multiple periods of heartbeat for it to settle into a steady periodic state. In addition, in order to calculate the viscous effect, $f$, using Eqs. (2) and (44), one needs the value of the blood flow rate, $Q$, for a full period. Therefore, it is rather expensive to calculate the viscous effect at each time step. In the traditional implementation of the Lax-Wendroff method for simulating pulse waves [15, 33], the viscous effect, $f$, of the full $(n+1)^{\text {th }}$ period is calculated using the flow rate, $Q$, of the $n^{\text {th }}$ period. In this sense, the traditional implementation of the Lax-Wendroff method can also be regarded as an iterative method from one period to the next to satisfy the periodic boundary condition. However, under this treatment, we observed that the solution may not always converge to a periodic solution with sufficient accuracy according to our simulation. We have found that it has a better convergent property if we average the viscous effect using a few periods. This average is implemented in the traditional method in the following when we compare the simulation runtime and calculate the speedup ratio.

The main aim of our algorithm is to overcome the constraint of small time step. A possible approach would be the employment of an adaptive mesh to reduce the simulation cost. However, to satisfy the CFL condition locally, a small time step may still be required in the simulation of many of the vessels. In addition, when the wave speed increases due to the vessel wall thickening and stiffening, such as in hypertension patients or aged people, the simulation cost also increases accordingly. In the following, using the formulations encapsulated in Section 2, we detail our numerical algorithm, in which the large wave speed will become a benefit instead of a constraint. 


\subsection{The fast algorithm based on spatial-temporal coordinate exchange}

In our approach, the spatial and temporal coordinates are exchanged in the following sense, namely, we rewrite Eq. (6) as

$$
\frac{\partial \Re^{ \pm}}{\partial x}+\frac{1}{u \pm c} \frac{\partial \Re^{ \pm}}{\partial t}=\frac{f}{\rho(u \pm c)},
$$

we then apply the Lax-Wendroff method by regarding $x$ as the "temporal" coordinate and $t$ as the "spatial" coordinate. After the coordinate exchange, the CFL condition is no longer a severe constraint in the case of large wave speed. In Eq. (23), the step length in $x$ determined by a given step length in $t$ can now be relatively large. In an iteration step in our algorithm, the value of $\Re^{+}$in each single vessel is updated using the Lax-Wendroff method starting with the "initial" value at the inlet of the vessel, whereas $\Re^{-}$is updated with its "initial" value at the outlet. According to Eqs. (2) and (4), the fast Fourier transform is used to calculate the viscous effect $f$ on the right hand side of Eq. (23). We note that in the exchanged coordinate system the periodic boundary condition (22) in time can be trivially realized in any finite difference method by setting the value of the right neighbor of the end "spatial" point to that of the first "spatial" point.

Our remaining task is to design an iterative method to enforce the spatial boundary conditions (12), (14), (18), and (21) at the inlet, the outlets, and the bifurcation points of the arterial tree, respectively. In our algorithm, each iteration step can be accomplished by updating $\Re^{+}$and $\Re^{-}$in the arterial tree by a level order traversal:

- Using Eq. (12) and the current value of $\Re_{\text {root }}^{-}(0, t)$ to update $\Re_{\text {root }}^{+}(0, t)$ at the inlet of the arterial tree (1 in Fig. 4 ). With the updated "initial value" $\Re_{\text {root }}^{+}(0, t)$, the Lax-Wendroff method for Eq. (23) is used in the exchanged-coordinate system to update $\Re_{\text {root }}^{+}(x, t)$ in the entire vessel $(2$ in Fig. (4);

- At each bifurcation point, when the value of $\Re_{p}^{+}\left(L_{p}, t\right)$ is updated, $\Re_{p}^{+}\left(L_{p}, t\right)$ and the current value of $\Re_{l}^{-}(0, t)$ and $\Re_{r}^{-}(0, t)$ of the two daughter vessels 


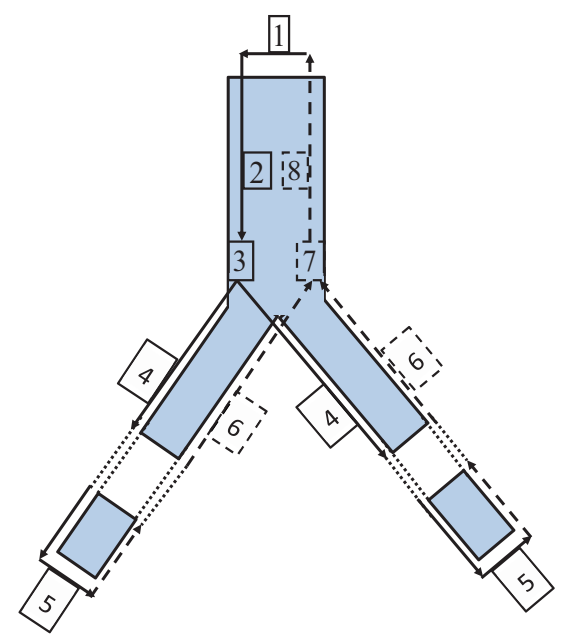

Figure 4: Schematics of our algorithm for an arterial tree. The numbers refer to the sequence of the traversal. The arrows indicate the direction of the traversal.

are substituted into the right hand side of Eq. (18) to update $\Re_{l}^{+}(0, t)$ and $\Re_{r}^{+}(0, t)$ at the bifurcation point $(3$ in Fig. 4$)$. Then $\Re_{l}^{+}(0, t)$ and $\Re_{r}^{+}(0, t)$ are used as the "initial values" to update the value of $\Re^{+}(x, t)$ in the two daughter vessels (4 in Fig. 4);

- At each outlet of the truncated arterial tree, the updated value of $\Re^{+}(L, t)$ is substituted into the right hand side of Eq. (14) to update the value of $\Re^{-}(L, t)$ at the outle(5 in Fig. 4 4 t, which is then used as the "initial value" to update $\Re^{-}(x, t)$ in the entire vessel (6 in Fig. 4);

- At each bifurcation point, when the values of $\Re_{l}^{ \pm}(0, t)$ and $\Re_{r}^{ \pm}(0, t)$ are updated, they are used to calculate the nonlinear reflection coefficient at the bifurcation point and Eq. (21) is used to update the value of $\Re_{p}^{-}(L, t)$ of the parent vessel at the bifurcation point ( 7 in Fig. 44), which is then used as the "initial value" to update $\Re_{p}^{-}(x, t)$ in the entire vessel 8 in Fig. (4).

In the application of the boundary conditions, transforms between the Riemann variables $\Re^{ \pm}$and $£^{ \pm}$are used whenever necessary. It is important to point 
out that the above iteration procedure can also be accomplished recursively. In what follows, we list the pseudocode of the recursive function "RECURSE $(V$, $\left.\Re_{i n}^{+}\right)$", where $V$ is the index of a vessel and $\Re_{i n}^{+}$is the input value of $\Re^{+}$at the inlet of vessel indexed by $V$. In the pseudocode, "in" and "out" in the subscript represent the inlet and outlet, respectively. Each iteration step is carried out by updating $\Re_{\text {root }}^{+}(0, t)$ with Eq. (12) then performing the recursive process at the root vessel.

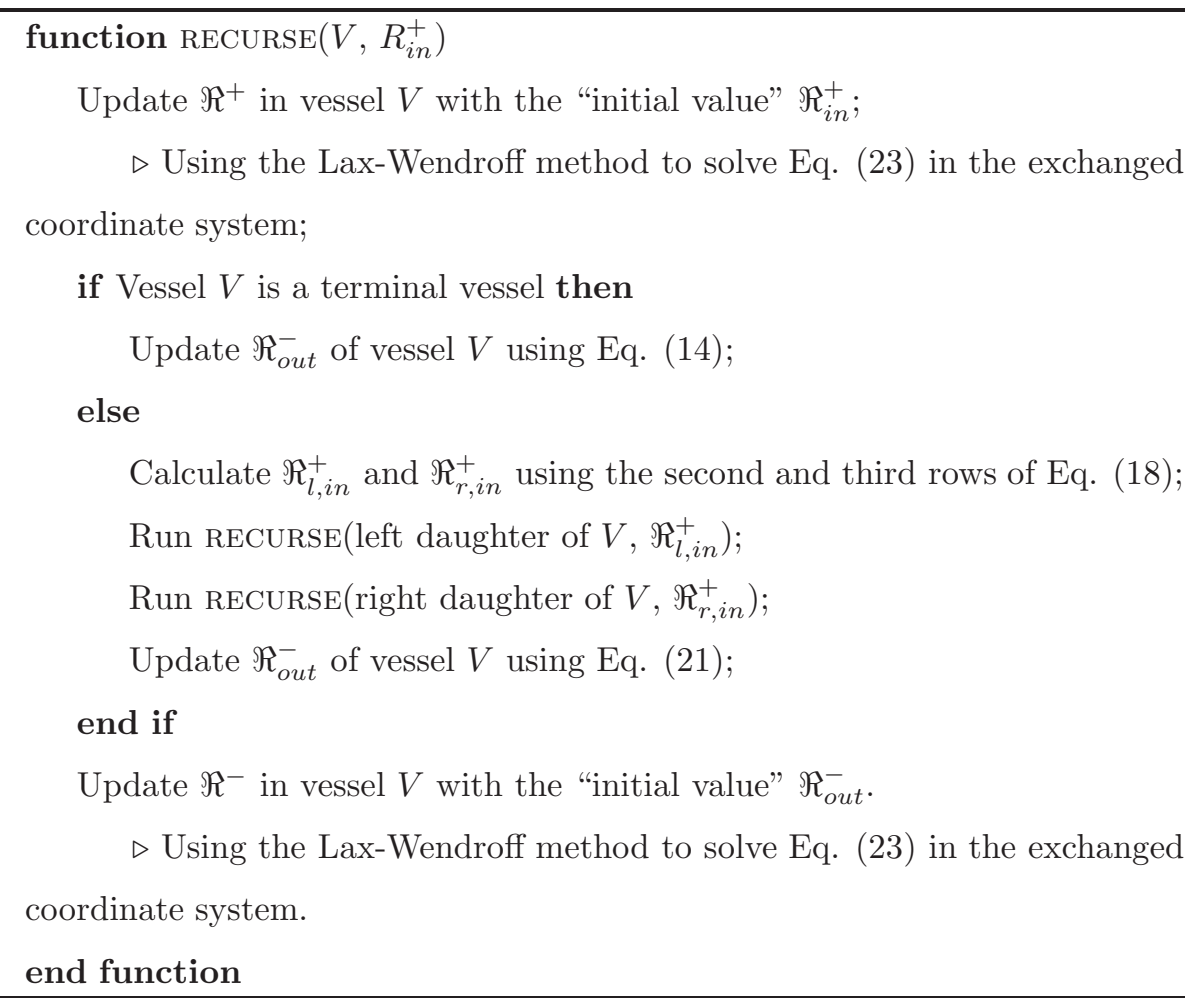

\section{Numerical Results}

The geometric data of the arterial tree, including the lengths, $L$, and the reference radii, $r_{0}$, of all the vessels, are tabulated in Table 4 which is adapted from Ref. [38]. The cardiac output $q_{c}(t)$ is obtained from Ref. [54]. The reflection coefficients at the outlets are computed with the structured tree model 
as discussed above [44]. The initial value of the Riemann variables can be trivially set to be zero.

Due to the difficulty of invasive measurement of blood pressure, there are few works that have evaluated the modeling error of one-dimensional models of blood pulse waves. However, simulation results have been compared with the measurements from a network of silicone vessels [23], in which the modeling error of the one-dimensional models is $\sim 4 \%$ for blood pressure and $\sim 19 \%$ for blood flow. In real applications, the uncertainty in the geometric and elastic properties of the arterial network as well as the uncertainty in the inflow boundary conditions can also give rise to modeling errors. A reasonable requirement is that the numerical error in a simulation be much smaller than the modeling error, e.g., smaller than one percent.

There are two sources of the numerical error in our algorithm - the spatial and temporal discretization error and the iterative convergence error. In the following, we first address the convergency of the iterative method, then discuss the effect of the discretization error, and finally compare the results from the simulations of the full system of the arterial tree (shown in Fig. 2A) using our numerical algorithm with that from the traditional implementation of the Lax-Wendroff method.

\subsection{Convergence of our algorithm}

In Fig. 5, we show the convergence of relative errors of the two Riemann variables and the blood pressure and flow rate over each iteration step. Here the convergence error of a function $g$ is defined as

$$
E_{g}(n)=\frac{1}{2 N_{V}} \sum_{i=1}^{N_{V}}\left(\frac{\left\|g_{i, n}(0, t)-g_{i}(0, t)\right\|_{2}}{\left\|g_{i}(0, t)\right\|_{2}}+\frac{\left\|g_{i, n}\left(L_{i}, t\right)-g_{i}\left(L_{i}, t\right)\right\|_{2}}{\left\|g_{i}\left(L_{i}, t\right)\right\|_{2}}\right),
$$

where $N_{V}$ is the total number of vessels in the arterial system, $i$ is the vessel index, $g_{i}(x, t)$ is the well converged numerical value of $g$, and $g_{i, n}(x, t)$ is the corresponding value of $g$ at the $n^{\text {th }}$ iteration step. As can be seen from Fig. 5. the convergence of the iteration algorithm is of first order and we only need approximately 17 iterations to obtain a relative error of the blood pressure 


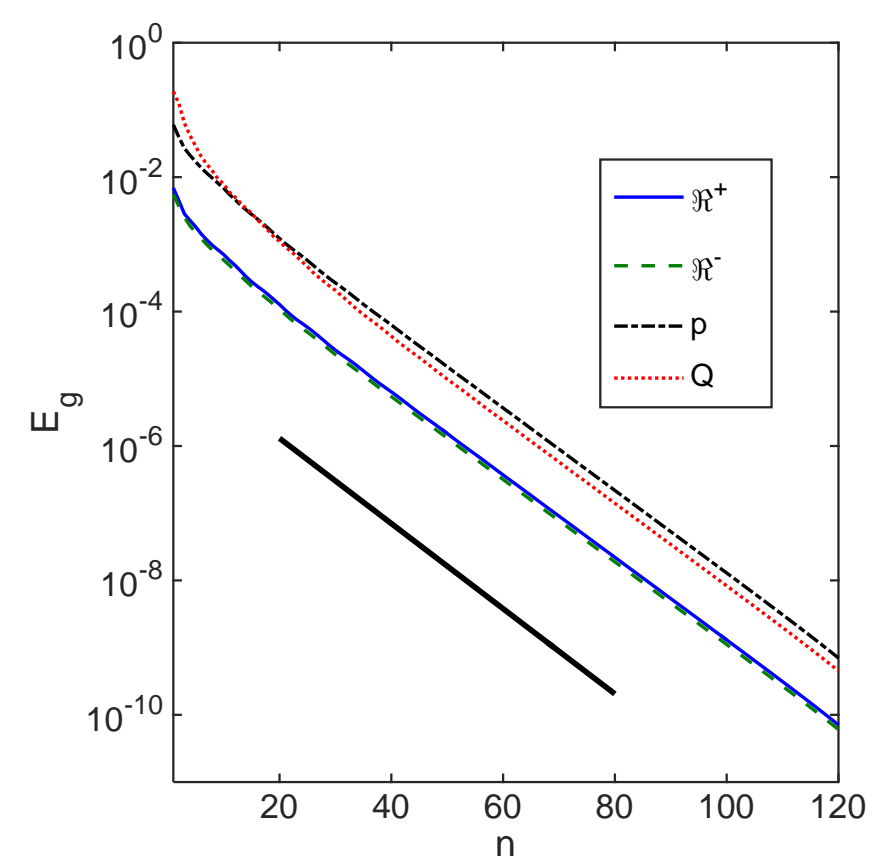

Figure 5: The convergence test. Shown here is the error of each iteration step as defined in Eq. (24) for the Riemann variables $\Re^{+}$(blue solid line) and $\Re^{-}$(green dashed line), the pressure $p$ (black dash-dot line), and the flow rate $Q$ (red dotted line). For comparison, the black solid line has a slope of $\log _{10}(0.86) \approx-0.066$, where 0.86 is the convergence rate.

smaller than one percent. This iteration number is about twice that in the traditional implementation of the Lax-Wendroff method with similar numerical accuracy. Incidentally, we observe that the numerical error of the blood pressure and flow rate is typically about one order of magnitude greater than that of the wo Riemann variables. In the following, we discuss how the error can propagate in the recursively updating process. Intuitively, this discussion can provide insight into the convergence of the iterative algorithm.

First, we discuss the propagation of the numerical error of the Riemann variables in a single vessel $(2,4,6,6$, and 8 in Fig. 44). Due to the weak nonlinearity of the system, the interaction between waves of different frequencies 
is weak. This results in the propagation of the error of different frequencies almost separated from each other. As can be seen from Eq. (4), the viscous effect $f$ is small for low-frequency components and large for high-frequency components. The viscous effect induces a decay in the amplitude of the waves. Therefore, the error of low-frequency components can propagate in the Riemann variables whereas the error of high-frequency components will eventually decay.

Next, we discuss the error transmission at the boundaries by using the boundary conditions (12), (14), (18), and (21) in "RECURSE". At the inlet of the root vessel, the numerical error of $\left(\Re^{-}\right)^{2}$ is directly passed to $\left(\Re^{+}\right)^{2}$ through Eq. (12) in each iteration step (1 in Fig. 4). At the outlets of the vessel tree (5 in Fig. 4), as the reflection ratio in Eq. (14) is always smaller than 1 , the numerical error in $£^{+}$is reduced when passed to $£^{-}$. In particular, the error of high-frequency components can reduce significantly through iteration.

At a bifurcation point, there is a similar reduction in the numerical error of the Riemann variables by applying Eq. (21) (4 in Fig. 4). It is worth noting that the numerical error in the reflection coefficient in Eq. (21) can also contribute to the numerical error of $£^{-}$. However, due to the weak nonlinearity, the difference between the linear and nonlinear reflection coefficients are not very large for low-frequency components (as shown in Fig. 3). As a result, the numerical error is still reduced in the iteration for low frequency components. Note that the reduction in the numerical error of high-frequency components is always significant from Eq. (14). Therefore, the numerical error of both low-frequency and high-frequency components can be reduced by Eqs. (14) and (21).

As mentioned above, the application of Eq. (18) in the iteration could lead to an amplification of numerical error in the sense of $L^{\infty}$ norm $(3$ in Fig. 4). However, this amplification is usually not strong and it can be sufficiently compensated by the error reduction by Eqs. (14) (5 in Fig. 4) and (21) 7 in Fig. 4). As a result, the iteration based on the recursive update process becomes convergent. 


\subsection{Numerical accuracy}

The numerical solutions of the two Riemann variables $\Re^{ \pm}$, the blood pressure $p$, and the flow rate $Q$ of the representative vessels are displayed in Fig. 6. As can be seen from Fig 6, the curves obtained from our algorithm with the time step $\Delta t=T / 64$, where $T$ is the period of one heartbeat, overlap well with those obtained with $\Delta t=T / 512$. For the simulation results in Figs. 6 and 7 the space step in a vessel is chosen to be $\Delta x=L / n$, where $L$ is the length of the vessel and $n$ is the smallest integer allowed by the CFL condition. The spatial and temporal discretization errors of the Lax-Wendroff method are comparable.
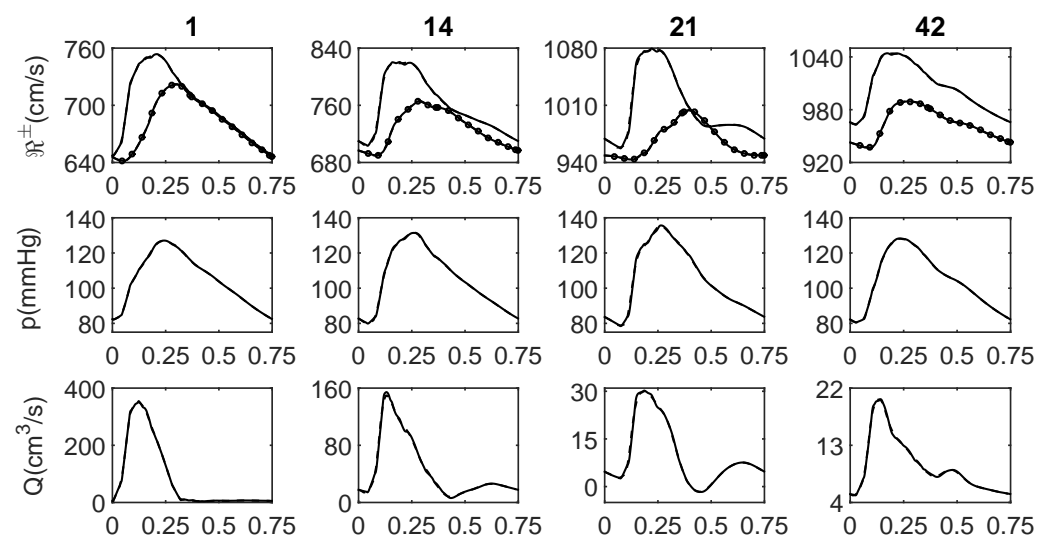

$\mathrm{t}(\mathrm{s})$

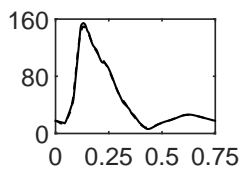

$\mathrm{t}(\mathrm{s})$

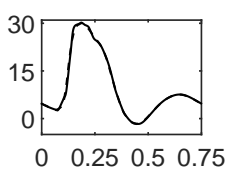

$\mathrm{t}(\mathrm{s})$

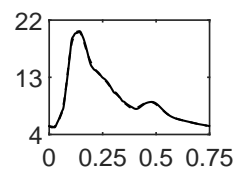

$\mathrm{t}(\mathrm{s})$

Figure 6: Numerical solutions of the two Riemann variables, the blood pressure, and the flow rate obtained from our algorithm. The dashed and solid lines stand for results obtained from the simulation with a time step length of $\Delta t=T / 64$ and $\Delta t=T / 512$, respectively, where $T$ is the period of heartbeat. In the upper panels, the quantity of $\Re^{-}$is marked with circles and the curves of $\Re^{+}$is not marked. The curves obtained from different sizes of time step overlap well with one another. The $x$-axis in each plot is the time in seconds over one period. The numbers on top of each column are the indices of arteries in Table 4 The values of the curves are obtained at the inlets of the corresponding vessels.

Using the well-converged solution obtained from our algorithm with $\Delta t=$ $\Delta_{0} \equiv T / 512$ as the reference solution, we can calculate the relative numerical error of solutions obtained from our algorithm and the traditional implementation of the Lax-Wendroff method. Here the relative numerical error of a function $g$ 

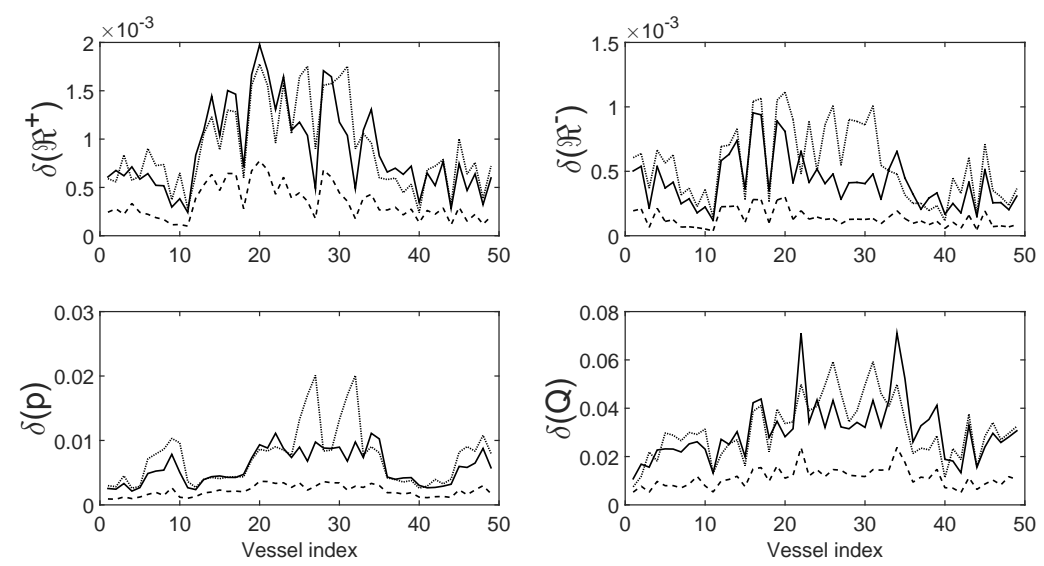

Figure 7: The relative error of $\Re^{+}, \Re^{-}, p$ and $\mathrm{Q}$ as defined in Eq. 25]. The solid line and the dashed line are obtained using our algorithm with $\Delta t=T / 64$ and $\Delta t=T / 128$, respectively. The dotted lines are obtained using the traditional method with $\Delta t=T / 2048$ and with the same space step as that used in the case of $\Delta t=T / 64$ in our algorithm.

at vessel $i$ is defined as

$$
\delta(g, \Delta t, i)=\frac{\left\|g_{i, \Delta t}\left(L_{i}, t\right)-g_{i, \Delta_{0}}\left(L_{i}, t\right)\right\|_{2}}{\left\|g_{i, \Delta_{0}}\left(L_{i}, t\right)\right\|_{2}},
$$

where $g_{i, \Delta t}$ and $g_{i, \Delta_{0}}$ are the value of $g$ in vessel $i$ obtained from the full simulation of the one-dimensional model with time step length $\Delta t$ and $\Delta_{0}$, respectively. The numerical order of accuracy $m_{g}(i)$ for vessel $i$ is defined as

$$
m_{g}(i)=\log _{2} \frac{\left\|g_{i, \Delta t}\left(L_{i}, t\right)-g_{i, \Delta t / 2}\left(L_{i}, t\right)\right\|_{2}}{\left\|g_{i, \Delta t / 2}\left(L_{i}, t\right)-g_{i, \Delta t / 4}\left(L_{i}, t\right)\right\|_{2}} .
$$

The numerical errors of all the vessels are illustrated in Fig. 7 and it can be seen that the numerical error decreases with decreasing time step. From the numerical error shown in Fig. 7, it can be estimated that the second order accuracy of the Lax-Wendroff method is lost in our algorithm as shown in the middle panel of Fig. 8. This is caused by the Gibbs phenomenon - as shown in the solid line in the left panel of Fig. 8, there is a rapid sharp transition in the inflow function at the beginning of each heartbeat. When the inflow function is sufficiently smooth, e.g., the dashed line in the left panel of Fig. 8, a second order accuracy can be recovered as demonstrated in the right panel of Fig. 8 , 

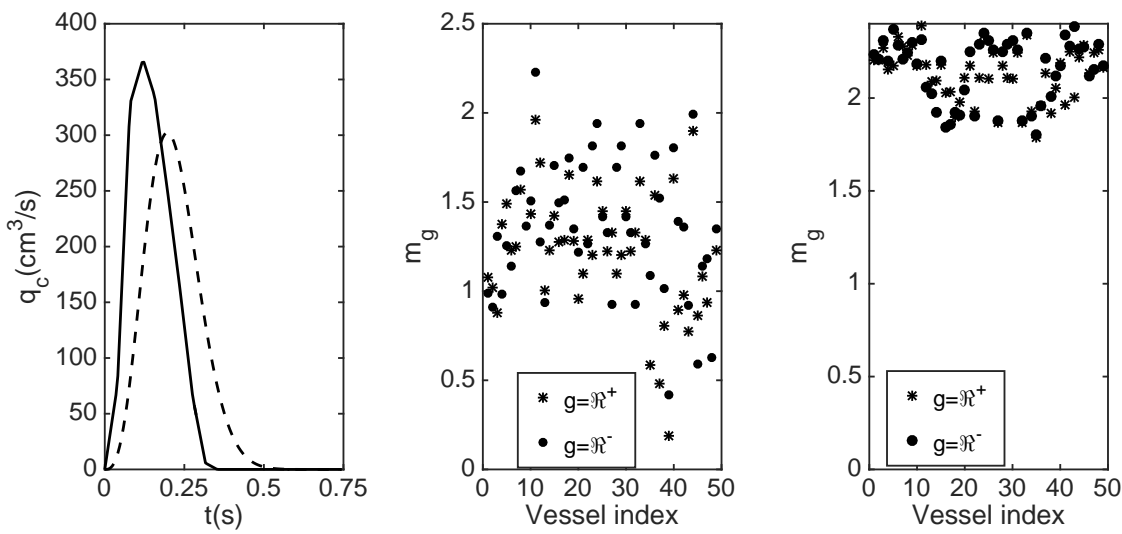

Figure 8: The accuracy order of the Lax-Wendroff scheme in our algorithm with different inflow rate $q_{c}(t)$. The nonsmooth inlet (solid line) in the left panel is derived from the experimentally measured blood flow rate in healthy people in the work of Ref [54] and the smooth inlet (dashed line) is a plot of the profile $q(t)=Q_{0} t^{3}(T-t)^{3} \exp \left(-25 t^{2}\right)$. The mean flow rates of the two profiles are $83.3 \mathrm{~cm}^{3} / \mathrm{s}$. The middle and the right panels show the accuracy order of $\Re^{ \pm}$with the nonsmooth inlet flow rate and the smooth inlet flow rate, respectively. The quantity of $\Re^{-}$is marked with circles and the result of $\Re^{+}$is marked with stars.

\subsection{Speed gain}

As can be seen from Fig. 7, using our algorithm, a time step of $T / 64$ is sufficient to reduce the relative numerical error smaller than one percent, which is already much smaller than the modeling error for blood pulse waves. In a two-core (Intel) PC with CPU rate 2.6GHz, the runtime for obtaining a solution of one period is 2.57 seconds using our algorithm with $\Delta t=T / 64$ and with the spatial step determined by the CFL condition. For a fare comparison, the space step of the traditional method is chosen to be the same as the new method. In order to satisfy the CFL condition, the time step of the traditional method is chosen to be $\Delta t=T / 2048$, which is nearly the largest $\Delta t$ allowed by the CFL condition. As shown in Fig. 7 the numerical error of the traditional method is comparable to that obtained with our algorithm. The runtime of the traditional method is 40.86 seconds. Thus, our algorithm can achieve about 15 times of speedup under the requirement that the relative numerical error of 
blood pressure be smaller than one percent.

\section{Summary and discussion}

In this work, we have developed a fast algorithm for obtaining periodic solutions of blood pulse waves in large arterial trees. By exchanging the spatial and temporal coordinates, we avoid the small time step constrained by the CFL condition due to the large wave speed. An efficient iteration method has been designed to satisfy the spatial boundary conditions. With the requirement that the relative numerical error of blood pressure be smaller than one percent, which is significantly smaller than the modeling error, our algorithm can perform 15 times faster than the traditional implementation of the Lax-Wendroff method. We believe that our work can provide a useful tool for future studies such as uncertainty quantification of the models of blood pulse wave and the inverse problem that relates blood pulse profiles to the characteristics in the geometric and elastic properties of the arterial tree.

In the previous modelling works, there have been discussions on the possible effects of vessel tapering [38] and the contribution of a convection term that comes from the deviation of the actual blood velocity from a flat profile in a cross section [15]. For the sake of description of the numerical algorithms, we have neglected these effects in this work. However, they can be easily incorporated in our method. As in the works [55, 56], the viscoelastic state equation between $p(x, t)$ and $A(x, t)$ can be written as

$$
p(x, t)=G(A(x, t))+\int_{0}^{t} E(x, t-\tau) A(x, \tau) d \tau
$$

or

$$
p(x, t)=G(A(x, t))+\eta \frac{\partial A(x, t)}{\partial t} .
$$

where $G(A(x, t))$ represents the elasticity, and the second term on the right hand side of both equations represents the memory effect. Both the two state equations have the form of

$$
p(x, t)=G(A(x, t))+F(A(x, \cdot)) .
$$


Therefore, we can rewrite the one-dimensional model as

$$
\begin{aligned}
\rho\left(u_{t}+u u_{x}\right)+\left.\frac{d G(x)}{d x}\right|_{x=A(x, t)} A_{x} & =f^{\prime}, \\
A_{t}+u A_{x}+A u_{x} & =0,
\end{aligned}
$$

${ }_{422}$ where $f^{\prime}=f-\frac{\partial F(\bar{A}(x, t))}{\partial x}$. Since the viscoelasticity does not discernibly affect the blood pulse wave propagation in arteries [57], our new algorithm can be applied similarly. In this work, we have focused our interest on the numerical method for periodic solutions of one heartbeat. Heartbeat may not be precisely periodic. If we have a multi-heartbeat measurement of the cardiac output function, $q_{c}(t)$, in $[0, n T]$, we can also incorporate this measurement in our method by setting $n T$ as the period of the periodic solution. In the periodic case, the traditional Lax-Wendroff method will be run 4 iterations to get a periodic solution. While there is no need to iterate the Lax-Wendroff method in the non-periodic case, our new method can still be 4 times faster than the traditional Lax-Wendroff method. When a parallel simulation is required, a Jacobi-like iteration method may be more efficient. That is, the Riemann variables are updated in the spatialtemporal exchanged space simultaneously in all vessels using Eq. (23). Then the reformulated boundary conditions Eq. (12), Eq. (14), Eq. (18), and Eq. (21) are used to update the Riemann variables at the ends of all vessels simultaneously. With the Jacobi-like iteration method, the new algorithm can achieve about 7 times speedup compared to the traditional Lax-Wendroff method. When the Windkessel models are used as the outflow boundary conditions, the differential form can lead to reduction of the time cost of the traditional Lax-Wendroff method by about $50 \%$ compared to the integral form. Thus the traditional method can be about 1.5 times faster for Windkessel boundary conditions than structured tree boundary conditions and our new method is about 10 times faster than the traditional method. 


\section{Acknowledgement}

This work was supported by grants NSFC 91230202, 11471213, 11071161, 31571071, and NSF DMS-1009575, Shanghai 14JC1403800, 15JC1400104, and the NYU Abu Dhabi Institute under grant G1301.

\section{References}

[1] M. Porkert, The essentials of Chinese diagnostics, Acta Medicinae Sinensis Zurich, 1983.

[2] L. I. Conrad, The Western Medical Tradition: 800 BC to AD 1800, Vol. 1, Cambridge University Press, 1995.

[3] J. Blacher, R. Asmar, S. Djane, G. M. London, M. E. Safar, Aortic pulse wave velocity as a marker of cardiovascular risk in hypertensive patients, Hypertension 33 (1999) 1111-1117. doi:10.1161/01.hyp.33.5.1111.

[4] F. N. van de Vosse, N. Stergiopulos, Pulse wave propagation in the arterial tree, Annual Review of Fluid Mechanics 43 (2011) 467-499. doi:10.1146/annurev-fluid-122109-160730

[5] N. Stergiopulos, D. F. Young, T. R. Rogge, Computer simulation of arterial flow with applications to arterial and aortic stenoses, Journal of Biomechanics 25 (12) (1992) 1477-1488. doi:10.1016/0021-9290(92)90060-e.

[6] H. Lax, A. W. Feinberg, B. M. Cohen, Studies of the arterial pulse wave: I. the normal pulse wave and its modification in the presence of human arteriosclerosis, Journal of Chronic Diseases 3 (6) (1956) 618-631. doi: 10.1016/0021-9681(56)90158-8

[7] G. E. McVeigh, C. W. Bratteli, D. J. Morgan, C. M. Alinder, S. P. Glasser, S. M. Finkelstein, J. N. Cohn, Age-related abnormalities in arterial compliance identified by pressure pulse contour analysis aging and arterial compliance, Hypertension 33 (6) (1999) 1392-1398. doi:10.1161/01.hyp.33.6.1392. 
[8] H. Lax, A. W. Feinberg, W. Urban, Abnormalities of the arterial pulse wave in young diabetic subjects, Circulation 20 (6) (1959) 1106-1110. doi:10.1161/01.cir.20.6.1106.

[9] W. Nichols, M. O'Rourke, C. Vlachopoulos, McDonald's blood flow in arteries: theoretical 6th Edition, experimental and clinical principles, CRC Press, 2011. doi:10.1201/b13568

[10] C. D. A. Stehouwer, R. M. A. Henry, I. Ferreira, Arterial stiffness in diabetes and the metabolic syndrome: a pathway to cardiovascular disease, Diabetologia 51 (4) (2008) 527-539. doi:10.1007/s00125-007-0918-3

[11] T. Christensen, B. Neubauer, Increased arterial wall stiffness and thickness in medium-sized arteries in patients with insulindependent diabetes mellitus, Acta Radiologica 29 (3) (1988) 299-302. doi:10.1177/028418518802900308

[12] T. Shoji, M. Emoto, K. Shinohara, R. Kakiya, Y. Tsujimoto, H. Kishimoto, E. Ishimura, T. Tabata, Y. Nishizawa, Diabetes mellitus, aortic stiffness, and cardiovascular mortality in end-stage renal disease, Journal of the American Society of Nephrology 12 (10) (2001) 2117-2124.

[13] M. O'Rourke, Mechanical principles in arterial disease, Hypertension 26 (1) (1995) 2-9. doi:10.1161/01.hyp.26.1.2.

[14] G. A. Johnson, H. S. Borovetz, J. L. Anderson, A model of pulsatile flow in uniform deformable vessel, J. Biomechanics 25 (1) (1992) 91-100. doi:10.1016/0021-9290(92)90248-y.

[15] K. Azer, C. S. Peskin, A one-dimensional model of blood flow in arteries with friction and convection based on the womersley velocity profile, Cardiovascular Engineering 7 (2) (2007) 51-73. doi:10.1007/s10558-007-9031-y. 
[16] T. J. R. Hughes, J. Lubliner, On the one-dimensional theory of blood flow in the larger vessels, Mathematical Biosciences 18 (1-2) (1973) 161-170. doi: 10.1016/0025-5564(73)90027-8

[17] A. C. L. Barnard, W. A. Hunt, W. P. Timlake, E. Varley, A theory of fluid flow in compliant tubes, Biophysical Journal 6 (6) (1966) 717-724. doi:10.1016/s0006-3495(66)86690-0

[18] Y. Huo, G. S. Kassab, Pulsatile blood flow in the entire coronary arterial tree: theory and experiment, American Journal of Physiology - Heart and Circulatory Physiology 291 (2006) H1074-H1087. doi:10.1152/ajpheart.00200.2006

[19] Y. Huo, G. S. Kassab, A hybrid one-dimensional/womersley model of pulsatile blood flow in the entire coronary arterial tree, American Journal of Physiology - Heart and Circulatory Physiology 292 (2007) H2623-H2633. doi:10.1152/ajpheart.00987.2006

[20] S. J. Sherwin, V. Franke, J. Peiró, K. Parker, One-dimensional modelling of a vascular network in space-time variables, Journal of Engineering Mathematics 47 (3/4) (2003) 217-250. doi:10.1023/b:engi.0000007979.32871.e2.

[21] D. Xiu, S. J. Sherwin, Parametric uncertainty analysis of pulse wave propagation in a model of a human arterial network, Journal of Computational Physics 226 (2) (2007) 1385-1407. doi:10.1016/j.jcp.2007.05.020

[22] J. Alastruey, K. H. Parker, S. J. Sherwin, Arterial pulse wave haemodynamics, in: Anderson. 11th International Conference on Pressure Surges, 2012, pp. 401-442.

[23] K. S. Matthys, J. Alastruey, J. Peiró, A. W. Khir, P. Segers, P. R. Verdonck, K. H. Parker, S. J. Sherwin, Pulse wave propagation in a model human arterial network: assessment of 1-d numerical simulations against 
in vitro measurements, Journal of Biomechanics 40 (15) (2007) 3476-3486. doi:10.1016/j.jbiomech.2007.05.027.

[24] J. Wan, B. Steele, S. A. Spicer, S. Strohband, G. R. Feijo'o, T. J. R. Hughes, C. A. Taylor, A one-dimensional finite element method for simulation-based medical planning for cardiovascular disease, Computer Methods in Biomechanics \& Biomedical Engineering 5 (3) (2002) 195-206. doi:10.1080/10255840290010670

[25] B. N. Steele, J. Wan, J. P. Ku, T. J. R. Hughes, C. A. Taylor, In vivo validation of a one-dimensional finite-element method for predicting blood flow in cardiovascular bypass grafts, Biomedical Engineering, IEEE Transactions on 50 (6) (2003) 649-656. doi:10.1109/tbme.2003.812201

[26] J. P. Mynard, P. Nithiarasu, A 1d arterial blood flow model incorporating ventricular pressure, aortic valve and regional coronary flow using the locally conservative galerkin (LCG) method, Communications in Numerical Methods in Engineering 24 (5) (2008) 367-417. doi:10.1002/cnm.1117.

[27] L. Formaggia, D. Lamponi, A. Quarteroni, One-dimensional models for blood flow in arteries, Journal of Engineering Mathematics 47 (3-4) (2003) 251-276. doi:10.1023/b:engi.0000007980.01347.29.

[28] M. Saito, Y. Ikenaga, M. Matsukawa, Y. Watanabe, T. Asada, P.-Y. Lagrée, One-dimensional model for propagation of a pressure wave in a model of the human arterial network: Comparison of theoretical and experimental results, Journal of Biomechanical Engineering 133 (12) (2011) 121005. doi:10.1115/1.4005472,

[29] F. Y. Liang, S. Takagi, R. Himeno, H. Liu, Biomechanical characterization of ventricular-arterial coupling during aging: a multiscale model study, Journal of Biomechanics 42 (6) (2009) 692-704. doi:10.1016/j.jbiomech.2009.01.010. 
[30] V. Eck, J. Feinberg, H. Langtangen, L. Hellevik, Stochastic sensitivity analysis for timing and amplitude of pressure waves in the arterial system, International Journal for Numerical Methods in Biomedical Engineering 31 (4). doi:10.1002/cnm.2711.

[31] M. Willemet, P. Chowienczyk, J. Alastruey, A database of virtual healthy subjects to assess the accuracy of foot-to-foot pulse wave velocities for estimation of aortic stiffness, American Journal of Physiology-Heart and Circulatory Physiology 309 (4) (2015) H663-H675. doi:10.1152/ajpheart.00175.2015

[32] R. D. Richtmyer, A survey of difference methods for non-steady fluid dynamics, National Center for Atmospheric Research, 1963.

[33] M. S. Olufsen, C. S. Peskin, W. Y. Kim, E. M. Pedersen, A. Nadim, J. Larsen, Numerical simulation and experimental validation of blood flow in arteries with structured-tree outlfow conditions, Annals of Biomedical Engineering 28 (2000) 1281-1299. doi:10.1114/1.1326031.

[34] N. P. Smith, A. J. Pullan, P. J. Hunter, An anatomically based model of transient coronary blood flow in the heart, SIAM Journal on Applied Mathematics 62 (3) (2002) 990-1018. doi:10.1137/s0036139999355199.

[35] O. San, A. E. Staples, An improved model for reduced-order physiological fluid flows, Journal of Mechanics in Medicine and Biology 12 (03) (2012) 1250052. doi:10.1142/s0219519411004666

[36] L. M. Itu, C. Suciu, A. Postelnicu, F. Moldoveanu, Analysis of outflow boundary condition implementations for 1d blood flow models, in: E-Health and Bioengineering Conference (EHB), IEEE, 2011, pp. 1-4.

[37] P. R. Nisam, L. S. Binu, A. K. Sukesh, One dimensional modeling and computation of blood flow and pressure of a stented artery, in: 2009 3rd International Conference on Bioinformatics and Biomedical Engineering, IEEE, 2009, pp. 1-5. doi:10.1109/icbbe.2009.5163145. 
[38] M. S. Olufsen, Modeling the arterial system with referency to an anesthesta simulator, Phd thesis, Department of Mathematics, Roskilde University (1998).

[39] R. Courant, K. Friedrichs, H. Lewy, Über die partiellen differenzengleichungen der mathematischen physik, Mathematische Annalen 100 (1) (1928) 32-74. doi:10.1007/bf01448839.

[40] X. Wang, J.-M. Fullana, P.-Y. Lagrée, Verification and comparison of four numerical schemes for a 1d viscoelastic blood flow model, Computer Methods in Biomechanics and Biomedical Engineering 18 (15) (2014) 1704-1725. doi:10.1080/10255842.2014.948428.

[41] Y. He, H. Liu, R. Himeno, A one-dimensional thermo-fluid model of blood circulation in the human upper limb, International Journal of Heat and Mass Transfer 47 (12) (2004) 2735-2745. doi:10.1016/j.ijheatmasstransfer.2003.10.041.

[42] G. Rudinger, Reviews of current mathematical methods for the analysis of blood flow, Biomedical Fluid Mechanics Symposium, ASME. Denver, Colorado (1966) 1-33.

[43] L. A. Taylor, J. H. Gerrard, Pressure-radius relationships for elastic tubes and their application to arteries: Part 1-theoretical relationships, Journal of Medical and Biological Engineering 15 (1977) 11-17. doi:10.1007/bf02441569.

[44] M. S. Olufsen, Structured tree outflow condition for blood flow in larger systemic arteries, American Journal of Physiology - Heart and Circulatory Physiology 276 (1) (1999) H257-H268.

[45] J. R. Womersley, Method for the calculation of velocity, rate of flow and viscous drag in arteries when the pressure gradient is known, The Journal of Physiology 127 (3) (1955) 553-563. doi:10.1113/jphysiol.1955.sp005276. 
[46] N. Westerhof, N. Stergiopulos, M. I. M. Noble, Snapshots of hemodynamics: an aid for clinical research and graduate education, Springer Science \& Business Media, 2010. doi:10.1007/978-1-4419-6363-5.

[47] S. Oka, M. Nakai, Optimality principle in vascular bifurcation, Biorheology 24 (1987) 737-751.

[48] B. K. Podesser, F. Neumann, M. Neumann, W. Schreiner, G. Wollenek, R. Mallinger, Outer radius-wall thickness ratio, a postmortem quantitative histology in human coronary arteries, Acta Anatomica 163 (1998) 63-68. doi:10.1159/000046485.

[49] M. R. Mirzaee, O. Ghasemalizadeh, B. Firoozabadi, Simulating of human cardiovascular system and blood vessel obstruction using lumped method, World Academy of Science, Engineering and Technology 41 (2008) 366-374.

[50] J. J. Wang, K. H. Parker, Wave propagation in a model of the arterial circulation, Journal of Biomechanics 37 (4) (2004) 457-470. doi:10.1016/j.jbiomech.2003.09.007.

[51] B. S. Gow, An electrical caliper for measurement of pulsatile arterial diameter changes in vivo., Journal of Applied Physiology 21 (3) (1966) 1122-1126.

[52] W. W. Nichols, M. F. ORourke, A. P. Avolio, T. Yaginuma, J. P. Murgo, C. J. Pepine, C. R. Conti, Age-related changes in left ventricular/arterial coupling, in: Ventricular/Vascular Coupling, Springer, 1987, pp. 79-114. doi:10.1007/978-1-4613-8634-6_4

[53] T. Du, D. Hu, D. Cai, Outflow boundary conditions for blood flow in arterial trees, PLoS ONE 10 (5) (2015) e0128597. doi:10.1371/journal.pone.0128597

[54] R. J. Van der Geest, V. G. M. Buller, J. H. C. Reiber, Automated quantification of flow velocity and volume in the ascending and descending aorta using flow velocity mapping, in: Computers in Cardiology 1995, IEEE, 1995, pp. 29-32. doi:10.1109/cic.1995.482563. 
[55] D. Valdez-Jasso, M. Haider, H. Banks, D. B. Santana, Y. Z. Germán, R. L. Armentano, M. S. Olufsen, et al., Analysis of viscoelastic wall properties in ovine arteries, Biomedical Engineering, IEEE Transactions on 56 (2) (2009) 210-219. doi:10.1109/tbme.2008.2003093

[56] R. Raghu, I. E. Vignon-Clementel, C. A. Figueroa, C. A. Taylor, Comparative study of viscoelastic arterial wall models in nonlinear one-dimensional finite element simulations of blood flow, Journal of Biomechanical Engineering 133 (8) (2011) 081003. doi:10.1115/1.4004532.

[57] G. E. Saito, T. J. Vander Werff, The importance of viscoelasticity in arterial blood flow models, Journal of biomechanics 8 (3) (1975) 237-245. doi:10.1016/0021-9290(75)90030-5. 
Table 1: Geometrical data of the human systemic large arterial system

\begin{tabular}{|c|c|c|c|c|c|c|c|}
\hline Index & Artery Name & $\mathrm{L}(\mathrm{cm})$ & $r_{0}(\mathrm{~cm})$ & Index & Artery Name & $\mathrm{L}(\mathrm{cm})$ & $r_{0}(\mathrm{~cm})$ \\
\hline 1 & Ascending aorta & 3.00 & 1.440 & 26 & L. Post. tibial & 32.00 & 0.247 \\
\hline 2 & Aortic arch & 2.00 & 1.353 & 27 & L. Ant. tibial & 34.25 & 0.130 \\
\hline 3 & L. Com. carotid & 20.75 & 0.370 & 28 & R. Ext. iliac & 5.75 & 0.368 \\
\hline 4 & Aortic arch & 4.00 & 1.300 & 29 & R. Femoral & 14.50 & 0.347 \\
\hline 5 & L. Subclavian & 3.50 & 0.423 & 30 & R. Femoral & 44.25 & 0.299 \\
\hline 6 & L. Brachial & 42.25 & 0.403 & 31 & R. Post. tibial & 32.00 & 0.247 \\
\hline 7 & L. Ulnar & 6.75 & 0.215 & 32 & R. Ant. tibial & 34.25 & 0.130 \\
\hline 8 & L. Ulnar & 17.00 & 0.203 & 33 & R. Deep femoral & 12.50 & 0.255 \\
\hline 9 & L. Interosseus & 8.00 & 0.091 & 34 & R. Int. iliac & 5.00 & 0.200 \\
\hline 10 & L. Radial & 23.50 & 0.174 & 35 & Inf. mesenteric & 5.00 & 0.160 \\
\hline 11 & L. Vertebral & 14.75 & 0.188 & 36 & R. Renal & 3.25 & 0.260 \\
\hline 12 & Thoracic aorta & 5.25 & 1.194 & 37 & Celiac axis & 1.00 & 0.390 \\
\hline 13 & Thoracic aorta & 10.50 & 1.071 & 38 & Hepatic & 6.50 & 0.220 \\
\hline 14 & Abdominal aorta & 5.25 & 0.861 & 39 & Hepatic & 1.00 & 0.220 \\
\hline 15 & Sup. mesenteric & 6.00 & 0.435 & 40 & Intercostal & 8.00 & 0.200 \\
\hline 16 & Abdominal aorta & 1.00 & 0.772 & 41 & Brachiocephalic & 3.50 & 0.620 \\
\hline 17 & Abdominal aorta & 1.00 & 0.756 & 42 & R. Com. carotid & 17.75 & 0.370 \\
\hline 18 & L. Renal & 3.25 & 0.260 & 43 & R. Subclavian & 3.50 & 0.423 \\
\hline 19 & Abdominal aorta & 10.00 & 0.740 & 44 & R. Vertebral & 14.75 & 0.188 \\
\hline 20 & Abdominal aorta & 7.00 & 0.601 & 45 & R. Brachial & 42.25 & 0.403 \\
\hline 21 & L. Ext. iliac & 5.75 & 0.368 & 46 & R. Radial & 23.50 & 0.174 \\
\hline 22 & L. Int. iliac & 5.00 & 0.200 & 47 & R. Ulnar & 6.75 & 0.215 \\
\hline 23 & L. Femoral & 14.50 & 0.347 & 48 & R. Interosseus & 8.00 & 0.091 \\
\hline 24 & L. Deep femoral & 12.50 & 0.255 & 49 & R. Ulnar & 17.00 & 0.203 \\
\hline 25 & L. Femoral & 44.25 & 0.299 & & & & \\
\hline
\end{tabular}

The vessel index is used in Fig. 2 to label the vessel. $L$ and $r_{0}$ are the fixed length and unstressed radius of each vessel, respectively. The data are adapted from the geometrical data in Ref. 38]. 